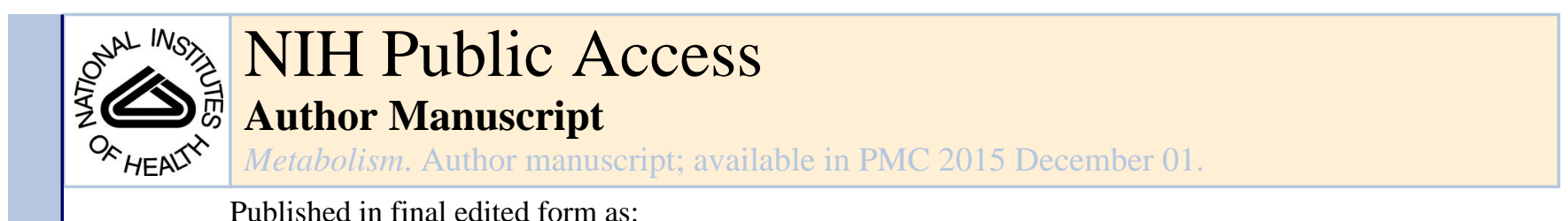

Published in final edited form as:

Metabolism. 2014 December ; 63(12): 1528-1535. doi:10.1016/j.metabol.2014.08.008.

\title{
Pre-diabetes in overweight youth and early atherogenic risk
}

\author{
Stephen F. Burns, Ph.D. ${ }^{1,3}$, SoJung Lee, Ph.D. ${ }^{1}$, Fida Bacha, M.D. ${ }^{4}$, Hala Tfayli, M.D. ${ }^{5}$, \\ Tamara S. Hannon, M.D. ${ }^{6}$, and Silva A. Arslanian, M.D. ${ }^{1,2}$ \\ ${ }^{1}$ Division of Weight Management and Wellness, Children's Hospital of Pittsburgh of UPMC \\ Pittsburgh, Pennsylvania 15201 \\ 2Division of Pediatric Endocrinology, Metabolism and Diabetes Mellitus Children's Hospital of \\ Pittsburgh of UPMC Pittsburgh, Pennsylvania 15201 \\ ${ }^{3}$ Physical Education and Sports Science Academic Group Nanyang Technological University, \\ Singapore 637616 \\ ${ }^{4}$ Children's Nutrition Research Center, Baylor College of Medicine, Houston, TX \\ ${ }^{5}$ Department of Pediatrics and Adolescent Medicine, American University of Beirut, Beirut, \\ Lebanon \\ ${ }^{6}$ Departments of Pediatrics, Indiana University School of Medicine, Indianapolis, IN
}

\section{Abstract}

Purpose-To compare atherogenic lipoprotein particles and vascular smooth muscle biomarkers in overweight youth with pre-diabetes (PD) vs. normal glucose tolerance (NGT).

\begin{abstract}
Methods-144 adolescents (60 black, 84 white; 102 female; PD=45, NGT=99) aged 10-19 years underwent a fasting blood draw and 2-hr OGTT. Lipoprotein particle size and subclass concentration and vascular smooth muscle biomarkers (ICAM-1, VCAM-1 and E-selectin) were compared between youth with PD and NGT.
\end{abstract}

\begin{abstract}
Results-Compared with NGT, PD adolescents had smaller LDL (mean \pm SE: $20.5 \pm 0.1 \mathrm{vs}$. $21.0 \pm 0.1 \mathrm{~nm} ; \mathrm{P}=0.002)$ and $\mathrm{HDL}(8.62 \pm 0.05$ vs. $8.85 \pm 0.04 \mathrm{~nm} ; \mathrm{P}=0.013)$ size and elevated medium small $(159.2 \pm 10.3$ vs. $123.8 \pm 6.4 \mathrm{nmol} / \mathrm{L} ; \mathrm{P}=0.037)$ and very small $(626.3 \pm 45.4$ vs. $458.5 \pm 26.4 \mathrm{nmol} / \mathrm{L} ; \mathrm{P}=0.032) \mathrm{LDL}$ particle concentrations, after adjustment for race and BMI. Further adjusting for fasting insulin or visceral adiposity obviated these differences between the groups except for LDL size. ICAM-1 and E-selectin did not differ in youth with PD but correlated with LDL and HDL size, and small LDL particle concentrations.
\end{abstract}

\section{(C) 2014 Elsevier Inc. All rights reserved}

Correspondence and requests for reprints: Silva Arslanian, MD Richard L Day Professor of Pediatrics Children's Hospital of Pittsburgh One Children's Hospital Drive 4401 Penn Avenue Pittsburgh, PA15224 Phone: (+001) 4126926565 Fax: (+001) 412692 6783 silva.arslanian@chp.edu.

Publisher's Disclaimer: This is a PDF file of an unedited manuscript that has been accepted for publication. As a service to our customers we are providing this early version of the manuscript. The manuscript will undergo copyediting, typesetting, and review of the resulting proof before it is published in its final citable form. Please note that during the production process errors may be discovered which could affect the content, and all legal disclaimers that apply to the journal pertain.

DISCLOSURE STATEMENT The authors have nothing to disclose. None of the authors have any conflict of interest to report with respect to this work. 
Conclusions-Overweight adolescents with PD have an atherogenic lipoprotein profile of small LDL and HDL size and increased concentrations of small LDL, moderated by insulin resistance and visceral adiposity, but independently driven by dysglycemia for LDL size. Associations between smooth muscle biomarkers and lipoproteins could be an early signal heralding the atherogenic process. It remains to be determined if correction of dysglycemia and associated lipoprotein abnormalities in obese youth could prove effective in halting this process.

\section{Keywords}

dyslipidemia; lipoproteins; glycemia; obesity; adolescents

\section{INTRODUCTION}

The transition from normal glucose tolerance (NGT) to overt type 2 diabetes mellitus is characterized by an intermediate state termed pre-diabetes (PD) which is representative of individuals with impaired fasting glucose (IFG) and impaired glucose tolerance (IGT) [1,2]. Data from the National Health and Nutrition Examination Survey (NHANES) in 2005-2006 found that the overall prevalence of PD in youth in the U.S. was $16.1 \%$ but that the prevalence was 1.6- and 2.6-fold higher in overweight and obese youth, respectively, compared with normal-weight children [3].

Overweight youth with PD often present with dyslipidemia including higher total cholesterol and low-density lipoprotein (LDL) cholesterol, higher triglycerides (TG) and lower highdensity lipoprotein (HDL) cholesterol [3,4,5]. However, traditional lipid measures only partially predict future cardiovascular disease risk $[6,7,8]$ and adult studies have found adverse lipoprotein particle size and subclass concentration in individuals with PD [9,10]. In youth, only one study [11] has examined the relationship between overweight, glycemia and atherogenic lipoprotein particles in 21 obese adolescents with PD (IFG and IGT) compared with 74 normoglycemic, obese counterparts. Despite similar standard lipid profiles, those youth with PD had smaller LDL and HDL particle size, higher concentrations of small LDL and HDL particles and lower concentrations of large HDL particles, the significance of all of which disappeared except for LDL particle size, after controlling for Homeostasis Model Assessment- Insulin Resistance Index (HOMA-IR) [11]. However, the authors highlighted that their study contained a relatively small number of youth with PD, which they suggest could lead to a type II error, and was largely represented by African Americans who made up $\sim 90 \%$ of the PD group and $\sim 78 \%$ of the normoglycemic youth [11]. Moreover, they were unable to examine the role of visceral adiposity in mediating the relationship between glycemia and atherogenic lipoprotein particles which has been highlighted in prior studies of youth $[12,13]$. Finally, circulating biomarkers of vascular smooth muscle function are increased in the early stage of vascular fatty lesions and play an important role in the formation of the atherosclerotic plaque [14] alongside lipoproteins. However, there has been no examination of these vascular biomarkers in relation to glycemia and PD in youth.

Thus, the aim of the present study was: 1) to compare differences in lipoprotein particle size and concentration in a large multi-racial (black/white) cohort of overweight adolescents with PD vs. NGT; 2) to examine the role of whole body and visceral adiposity in mediating 
differences in lipoprotein particle size and concentration between these two groups; and 3) to investigate differences in biomarkers of vascular smooth muscle function in overweight youth with PD vs. NGT.

\section{METHODS}

\subsection{Subjects}

Participants were 144 black and white overweight/obese (body mass index, BMI $\geq 85^{\text {th }}$ percentile) adolescents aged 10-19 years. For some participants data on lipids or lipoprotein particle size and subclass concentration were reported before but within a different context and specific aims, as part of a grant investigating childhood insulin resistance $[12,15,16,17,18]$. None of these previous studies examined the role of established clinical definitions of glycemia or PD in youth on lipoprotein particle size or subclass concentration or vascular smooth muscle markers. Study participants were recruited through newspaper and bulletin board advertisements. All studies were approved by the Institutional Review Board of the University of Pittsburgh. All participants and their parents gave written informed assent and consent after a thorough explanation of the proposed study. Exclusion criteria included diagnosed diabetes and the use of medications that influence glucose and lipid metabolism or blood pressure. These medications included oral contraceptive pills, metformin, anti-psychotic drugs, fish oils and drugs for dyslipidemia and hypertension. Participants' health was assessed by medical history, physical examination and routine hematological and biochemical tests. Pubertal development was assessed by physical examination according to Tanner criteria.

\subsection{Anthropometry}

All participants were admitted to the Children's Hospital of Pittsburgh National Institutes of Health funded Pediatric Clinical and Translational Research Center. Body height and weight were measured to the nearest $0.1 \mathrm{~cm}$ and $0.1 \mathrm{~kg}$, respectively, using standardized equipment.

\subsection{Body composition}

Total body fat was assessed using dual energy X-ray absorptiometry (DEXA). Abdominal subcutaneous and visceral adipose tissues (VAT) were determined from a single axial image (10-mm thickness) of the abdomen at the level of the L4-L5 intervertebral disc using computed tomography. Both methods have been described previously [19].

\subsection{Fasting blood draw and Oral glucose tolerance test (OGTT)}

After an overnight fast, blood samples were obtained for lipoprotein particle size and concentration and vascular smooth muscle biomarkers, followed with a 2-h OGTT (1.75 $\mathrm{g} / \mathrm{Kg}$ glucola, maximum $75 \mathrm{~g}$ ) in all participants. Blood samples were obtained at $-15,0,15$, 30, 60, 90 and 120 minutes for determination of glucose and insulin concentrations.

\subsection{Biochemical measurements}

Plasma glucose was measured using a glucose analyzer (YSI, Yellow Springs, OH) and insulin concentrations were measured by radioimmunoassay [15]. Plasma lipid 
concentrations (total, HDL and LDL cholesterol and total and very low density lipoprotein (VLDL)-TG) were determined using the standards of the Centers for Disease Control and Prevention as described previously [12]. For total and HDL cholesterol and total TG intraassay coefficients of variation (CV) were $1.0 \%, 1.8 \%$ and $1.8 \%$ and inter-assay CV $1.6 \%$, $2.6 \%$ and $3.7 \%$, respectively. LDL and VLDL were calculated using the Friedewald equation [20]. Concentrations of lipoprotein subclasses and particle size were determined using nuclear magnetic resonance (NMR) spectroscopy at LipoScience Inc. using the LipoProfile-2 algorithm (LipoScience Inc., Raleigh, NC) [21]. Using this method the quantity of each subclass is reported in particle concentration units (nanomoles of particles per liter for VLDL and LDL and micromoles per liter for HDL). The VLDL, LDL, and HDL were separated into 10 subclass categories: large VLDL (including chylomicrons) ( $>60 \mathrm{~nm}$ ), medium VLDL (35-60 nm), small VLDL (27-35 nm), intermediate-density lipoprotein (IDL) (23-27 nm), large LDL (21.2-23 nm), medium-small LDL (19.8 -21.2 nm), very small LDL (18 -19.8 nm), large HDL ( $8.8-13 \mathrm{~nm})$, medium HDL $(8.2-8.8 \mathrm{~nm})$, and small HDL $(7.3-8.2 \mathrm{~nm})$. Average lipoprotein particle sizes were computed as the sum of the diameter of each subclass multiplied by its relative mass percentage as estimated from the amplitude of its methyl NMR signal [12,21]. Intra-assay and inter-assay coefficients of variation were estimated from two pools of plasma, one with high TG and low HDL and the other with low TG and high HDL [22]. Both intra- and inter-assay CV were $\leq 4 \%$ for total VLDL, HDL and LDL particles and typically $\mathbf{6} \%$ for all subclass concentrations [22]. Intra- and inter-assay CV for HDL and LDL particle size were $<1 \%$ and for VLDL size $<3 \%$ [22]. Biomarkers of vascular smooth muscle function, intercellular adhesion molecule-1 (ICAM-1), vascular adhesion molecule-1 (VCAM-1) and E-selectin, were quantified using commercially available double-sandwich enzyme-linked immunoassays (R\&D Systems, Minneapolis, MN). Intra-assay CV for ICAM-1, VCAM-1 and E-selectin were 5.96\%, $4.91 \%$ and $6.78 \%$ and inter-assay CV $9.37 \%, 9.01 \%$ and $8.98 \%$, respectively.

\subsection{Statistical analysis}

Statistical procedures were performed using SPSS 21.0 for Windows (SPSS, Chicago, IL). To investigate the relationship of the category of glycemia with lipoprotein particle size and concentration and markers of vascular smooth muscle function, participants were divided into two groups: i) NGT and ii) PD. Individuals with NGT had both normal fasting and 2-hr glucose concentrations. Individuals with PD were those with either IFG or IGT which was defined as fasting plasma glucose between 5.6 and $6.9 \mathrm{mmol} / \mathrm{L}$ or plasma glucose between 7.8 and $11.0 \mathrm{mmol} / \mathrm{L}$ at $120 \mathrm{~min}$ of the OGTT, respectively [1]. Differences in categorical variables (sex, race and Tanner stage) were determined by Chi-square analysis. Normality was checked for all continuous variables using a Kolmogorov-Smirnoff test and differences in variables between groups determined using independent t-test or Mann Whitney test. Adjustments for race and different measures of adiposity (BMI, fat mass, percentage body fat and VAT) were made using ANCOVA with data for non-normally distributed variables $\log$ transformed beforehand. Further ANCOVA was used to adjust for race and BMI with fasting insulin. Stepwise multiple regression, in all participants combined, was used to assess the effect of category of glycemia (NGT vs PD) along with race, sex, age, Tanner stage (II-III or IV-V), adiposity (BMI or VAT) and fasting insulin on lipoprotein particle size and concentration. Relationships of vascular smooth muscle markers with lipoprotein 
size and subclass concentration were determined using Spearman rank correlations $\left(\mathrm{r}_{\mathrm{s}}\right)$ as data for vascular markers were not normally distributed. Data are presented as mean \pm standard error (SE). Significance was set at $\mathrm{P}<0.05$.

\section{RESULTS}

\subsection{Physical and metabolic characteristics}

Physical and metabolic characteristics of the participants by glycemic category are presented in Table 1. The groups were similar in age and development. All youth were Tanner stage II or greater. Distribution of the sexes was similar in both groups but with more females than males overall. There was a significant racial difference between NGT and PD, such that the group with PD had significantly more white than black youth. Adolescents with PD had significantly greater VAT, higher fasting and 2-h OGTT glucose concentrations, and higher fasting insulin compared with NGT but with no difference in $\mathrm{HbA}_{1 \mathrm{c}}$ concentrations. When Log VAT was adjusted for race the difference between the two groups remained significant $(\mathrm{P}=0.022)$. In the PD group, 9 youth had isolated IFG, 31 had isolated IGT and 5 had both IFG and IGT.

\subsection{Lipid concentrations}

Fasting lipid profiles determined by chemical analysis are presented in Table 1 . There was no difference in cholesterol or LDL-cholesterol between groups but youth with PD had significantly lower concentrations of HDL-cholesterol and higher concentrations of TG and VLDL-TG than youth with NGT. Differences in HDL remained after correcting for race and any measure of adiposity (BMI, $\mathrm{P}=<0.001$; fat mass, $\mathrm{P}=0.001$; Log percentage body fat, $\mathrm{P}=0.001$; $\log \mathrm{VAT}, \mathrm{P}<0.001)$. Including $\log$ fasting insulin $(\mathrm{P}=0.001)$ in the adjustment for race and BMI did not change the significant difference in HDL between PD and NGT groups. Differences in VLDL remained significant between the two groups when correcting for race and BMI $(\mathrm{P}=0.031)$ but not race and $\log \mathrm{VAT}(\mathrm{P}=0.086)$; or race, $\mathrm{BMI}$ and $\log$ insulin $(\mathrm{P}=0.11)$. There were no differences in TG between $\mathrm{PD}$ and NGT after correcting for race, adiposity and Log insulin.

\subsection{Lipoprotein particle size}

Figure 1 depicts LDL (Figure 1A) and HDL (Figure 1B) particle size in youth with NGT vs. PD. Both LDL and HDL particle size were significantly smaller in adolescents with PD. These differences remained significant in the LDL particle after adjusting for race and any measure of adiposity (BMI, fat mass, Log percentage body fat and Log VAT), but disappeared for HDL particle size after adjusting for $\log$ VAT ( $\mathrm{P}=0.096)$. Adjusting for race, BMI and Log fasting insulin did not change the significant difference between groups in the LDL particle but removed differences in the HDL particle between youth with PD and NGT. There was no difference in VLDL particle size between groups (NGT, $53.7 \pm 1.0 \mathrm{~nm}$ vs. $\mathrm{PD}, 55.5 \pm 1.5 \mathrm{~nm} ; \mathrm{P}=0.282$ ).

\subsection{Lipoprotein particle concentrations}

Figure 2 shows lipoprotein particle concentrations. Large LDL (Figure 2A) particle concentrations were lower whilst, conversely, medium small (Figure 2B) and very small 
(Figure 2C) LDL particle concentrations were higher in the youth with PD than those with NGT. These differences in the concentration of LDL particles remained after correcting for race and most measures of adiposity (BMI, fat mass or percentage body fat), but not race and Log VAT (large LDL, $\mathrm{P}=0.057$; medium small LDL, $\mathrm{P}=0.130$; very small LDL, $\mathrm{P}=0.111)$. Differences in LDL particles between groups also disappeared after correcting for race, BMI and Log insulin. Large HDL concentrations (Figure 2D) were lower in adolescents with PD than those with NGT but this difference disappeared after correcting for race and adiposity. There were no differences in medium (Figure 2E) or small (Figure 2F) HDL particle concentrations between groups. Large (PD, $4.2 \pm 0.6 \mathrm{nmol} / \mathrm{L} v s$. NGT, $2.4 \pm$ $0.3 \mathrm{nmol} / \mathrm{L} ; \mathrm{P}=0.002)$ and medium $(\mathrm{PD}, 21.5 \pm 2.1 \mathrm{nmol} / \mathrm{L} v s . \mathrm{NGT}, 15.6 \pm 1.2 \mathrm{nmol} / \mathrm{L}$; $\mathrm{P}=0.006)$ VLDL particle concentrations were higher in youth with PD than those with NGT, with no difference in small VLDL (PD, $30.9 \pm 2.0 \mathrm{nmol} / \mathrm{L} v s$. NGT, $28.2 \pm 1.5 \mathrm{nmol} / \mathrm{L}$; $\mathrm{P}=0.302$ ). Adjusting for Log fasting insulin along with race and $\mathrm{BMI}$ obviated differences in all VLDL particles between groups.

As IFG and IGT have distinct pathophysiologic etiologies we further compared the data among 9 youth with isolated IFG and 31 with isolated IGT. Against the backdrop of the few IFG subjects, there were no significant differences in lipoprotein size or subclass concentration between the two groups (data not shown). The significant differences between PD and NGT in LDL and HDL size, medium and small LDL, and large and small VLDL concentrations persisted when only youth with isolated IGT were compared with NGT adolescents (data not shown).

Evaluation based on HbA1C diagnostic categories (1) of PD (5.7 to < 6.5\%) (n=33) vs. normal $(<5.7 \%)(\mathrm{n}=111)$ revealed significantly lower large $(\mathrm{PD}, 2.2 \pm 0.6 \mathrm{nmol} / \mathrm{L} v s$. Normal, $3.2 \pm 0.3 \mathrm{nmol} / \mathrm{L} ; \mathrm{P}=0.012)$ and medium (PD, $13.2 \pm 2.1 \mathrm{nmol} / \mathrm{L} v s$. Normal, 18.7 $\pm 1.2 \mathrm{nmol} / \mathrm{L} ; \mathrm{P}=0.014$ ) VLDL particle concentrations in $\mathrm{PD}$ youth, but these differences disappeared after correcting for race and BMI. No other differences in lipoprotein particle size and concentration existed. Only 9 youth had an $\mathrm{HbA1C} \geq 6.0 \%$ preventing any further comparison between groups using an International Definition of PD (HbA1C 6.0-6.4\%) as has been done by others [23].

\subsection{Contribution of glycemia to lipoprotein particle size and concentration}

Category of glycemia together with Log fasting insulin, race and sex explained $24.3 \%$ of the variance in LDL particle size (Table 2) but did not predict HDL or VLDL size (data not shown). When BMI was replaced with VAT in the model, category of glycemia (partial $\mathrm{r}=$ -0.237, $\mathrm{P}=0.008$ ) predicted $24.6 \%$ of LDL particle size along with Log VAT, Log fasting insulin and Tanner stage. Category of glycemia was the sole predictor for $3.1 \%$ of large LDL particle concentrations (partial $\mathrm{r}=-0.175, \mathrm{P}=0.037$ ) and significantly predicted $18.4 \%$ of medium small and $18.3 \%$ of very small LDL particle concentration in combination with Log insulin and sex (Table 2). Substitution of BMI with VAT removed category of glycemia as a predictor of all LDL subclass. Category of glycemia did not predict HDL or VLDL particle concentrations. 


\subsection{Vascular smooth muscle biomarkers}

Concentrations of the vascular smooth muscle biomarkers, ICAM-1, VCAM-1, and Eselectin were not different between NGT and PD (Table 1). The ICAM-1 concentration correlated significantly with the LDL, HDL and VLDL particle size $\left(r_{\mathrm{s}}=-0.222, \mathrm{r}_{\mathrm{s}}=-0.213\right.$, $r_{s}=0.178$ respectively, $\mathrm{P}=<0.05$ ), with concentrations of large, medium small and very small LDL $\left(r_{\mathrm{s}}=-0.183, \mathrm{r}_{\mathrm{s}}=0.173, \mathrm{r}_{\mathrm{s}}=0.217\right.$, respectively, $\left.\mathrm{P}<0.05\right)$, and with large HDL particles and large VLDL and chylomicron particles $\left(r_{\mathrm{s}}=-0.247, \mathrm{r}_{\mathrm{s}}=0.236\right.$, respectively, $\left.\mathrm{P}<0.01\right)$. Similarly, E-selectin correlated with LDL and HDL particle size $\left(r_{\mathrm{s}}=-0.199, \mathrm{r}_{\mathrm{s}}=-0.189\right.$ respectively, $\mathrm{P}<0.05)$, with medium small and very small LDL particle concentrations $\left(\mathrm{r}_{\mathrm{s}}=\right.$ $0.176, r_{s}=0.190$ respectively, $\left.\mathrm{P}<0.05\right)$, and large HDL particle concentration $\left(r_{s}=-0.196\right.$, $\mathrm{P}=0.020)$. VCAM-1 did not correlate with lipoprotein size or concentration.

\section{DISCUSSION}

The present study demonstrates that overweight youth with PD have an atherogenic lipoprotein profile of small dense LDL and HDL particle size, and high concentrations of small LDL and large VLDL particles, and low concentrations of large HDL particles, compared with their NGT peers. Differences in the LDL particle size remained even after adjustment for various adiposity indices and a surrogate of insulin sensitivity suggesting an independent effect of hyperglycemia on LDL particle size. Our data confirm findings from a smaller previous study which showed that obese youth with PD ( $n=21)$, primarily black, have a significantly more atherogenic lipoprotein profile compared with their normoglycemic peers [11]. The present investigation extends and strengthens the previous findings by examining a much larger multi-racial cohort of youth with PD, and reveals a role of visceral adiposity in the observed lipoprotein differences between youth with PD and NGT except for LDL particle size where dysglycemia itself plays a role. Lastly, the pathological translation of this atherogenic profile in youth with PD was examined by measuring circulating biomarkers of vascular smooth muscle dysfunction, with significant relationships noted between these markers and LDL and HDL particle size.

In youth, data from the NHANES in 2005-2006 found that the overall prevalence of PD was $16.1 \%$ [3]. However, the prevalence in overweight (BMI $85^{\text {th }}-<95^{\text {th }}$ percentile, $18.3 \%$ ) and obese (BMI $\geq 95^{\text {th }}$ percentile, $30.0 \%$ ) adolescents was considerably greater than that of their normal weight counterparts (11.6\%) [3]. Importantly, PD in youth was also associated with an increased number of cardiometabolic risk factors including low HDL-cholesterol and high triglycerides [3]. Our data confirm that youth with PD exhibit a worse standard lipid profile than their normoglycemic counterparts and also have an atherogenic lipoprotein profile exemplified above. Even though the risk for development of cardiovascular disease in youth with PD is not known [5], the current findings are disturbing given that large prospective studies in adults with PD show an increased risk of all cause and cardiovascular mortality [24,25] and non-fatal cardiovascular events [26,27,28]. The increased risk is probably related to the poor lipoprotein subclass profile $[9,10]$ which has been shown to be associated with carotid intima media thickness in adults [29], and a strong predictor of cardiovascular disease [7,8]. Moreover, childhood LDL and HDL have been related to carotid intima media thickness and its progression in adulthood [30], with normal weight 
and obese youth with favorable lipoprotein concentrations having lower intima media thickness in adulthood than obese youth with unfavorable profiles [32]. Additionally, since early atherosclerotic plaque formation and changes in carotid intima media thickness begin in childhood and have been related to hyperglycemia [32,33], consideration should be given to early treatment of hyperglycemia and associated lipoprotein abnormalities in overweight youth with PD.

We previously demonstrated that, in normal weight and overweight otherwise healthy youth, in vivo clamp-measured insulin sensitivity [18] and waist circumference [12] are important determinants of an atherogenic lipoprotein profile. Similar observations were made by others using a variety of methodologies [3,13,34,35,36,]. Magge and colleagues [11] reported that controlling for HOMA-IR eliminated the differences in lipoproteins between pre-diabetic and normoglycemic obese youth except for small LDL particle size, but controlling for age, sex, race, Tanner stage and BMI did not abolish the lipoprotein differences between the two groups [11]. In the current study, the persistence of a difference in LDL particle size between PD and NGT youth, after correcting for visceral adiposity and fasting insulin or HOMA-IR (data not shown), together with the data of Magge and colleagues [11], strongly suggest an independent contribution of hyperglycemia per se to LDL particle size. Indirect support for this is the recent observation from the TODAY trial that glycosylated hemoglobin was directly related to LDL concentrations independent of BMI in youth with type 2 diabetes [37].

Circulating biomarkers of vascular smooth muscle function are increased in response to inflammation in the early stage of fatty lesions and play a role in the initial formation of the atherosclerotic plaque [14] beginning in childhood [28,38]. In the current study, there were no differences in the concentrations of ICAM-1, VCAM-1 and E-selectin between youth with and without PD. The ability of these indirect markers to differentiate early endothelial dysfunction in overweight youth with or without PD could be questioned, and more direct endothelial challenge tests may be needed to distinguish endothelial dysfunction in youth with PD. Alternatively, cytokine markers of arterial inflammation, such as interleukin-6 and components of its transsignalling system which have been shown to correlate with cellular adhesion molecules and arterial stiffness in adults with metabolic syndrome [39], may be better to characterize early endothelial function in youth. Nevertheless, in the present study ICAM-1 and E-selectin were associated with the size of both LDL and HDL particles, and LDL particle concentration suggesting a possible link between atherogenic lipoprotein particles and the initial stages of smooth muscle dysfunction and atherosclerosis. Whether such differences would evolve over time with persistence of dysglycemia, obesity and dyslipidemia remain to be investigated.

The classification of PD includes individuals with IFG or IGT, with significant debate over the years, the most recent just released in 2014, about the various definitions [40]. For the purpose of the present study, adolescents with PD were clustered together and represented obese youth with IFG, IGT or both. Since IFG and IGT are reported to have distinct pathophysiologic etiologies [2,41,42], we further sub-analyzed and compared youth with isolated IFG versus isolated IGT, but found no significant differences in the lipoprotein profiles between the two. The paucity of numbers however is a limitation preventing any 
conclusion and larger multi-center studies are needed to examine this issue further.

Additionally, both IFG and IGT can be transient states with poor reproducibility of the oral glucose tolerance test in youth and adults [40,43], and progression from IGT to diabetes is far from guaranteed in adults and youth $[44,45]$. Thus, longitudinal examination of changes in atherogenic lipoproteins in relation to persistent hyperglycemia in obese youth is needed.

There are a number of limitations to the present study. This is a cross-sectional evaluation of data amassed with no a priori power analysis. Thus, our numbers may be insufficient to preclude the possibility of a type II error when comparing NGT and PD youth. However, our study contains more than twice the number of youth with PD than the study by Magge and colleagues [11] and is confirmatory of their work. Collectively, these studies provide evidence on the important relationship of PD with lipoproteins in youth. Another potential limitation is that visceral adiposity, an important modulator of lipoprotein particle size [12], was larger in youth with PD vs. NGT. However, the significant difference in LDL particle size between PD and NGT persisted even after adjusting for visceral fat. Finally, the use of fasting insulin, or its inverse or HOMA, as surrogate estimates of insulin sensitivity, may perhaps be viewed as a limitation. However, our group has shown that these surrogate estimates correlate strongly with in vivo insulin sensitivity measured with the hyperinsulinemic-euglycemic clamp in youth with NGT, PD and diabetes [46], particularly when applied to large numbers.

\section{CONCLUSIONS}

In summary, the present study shows that overweight/obese youth with pre-diabetes exhibit an atherogenic lipoprotein profile of small dense LDL and small HDL in combination with increased concentrations of small LDL and large VLDL particles, and low concentrations of large HDL particles compared with their normoglycemic counterparts. Our data suggest that physicians screening or treating overweight youth with PD should look beyond traditional lipid measurements, particularly for LDL cholesterol, to enable a better assessment of early cardiovascular risk. While significant relationships exist between atherogenic particles and vascular smooth muscle biomarkers, the absence of differences in these biomarkers between pre-diabetes and NGT provides hope that correction of dysglycemia, obesity and the lipoprotein abnormalities at this early stage might prevent the genesis of atherosclerosis.

\section{Acknowledgments}

We express our gratitude to all the volunteer children and their parents without who science could not be advanced. We are grateful to the nursing staff of the Pediatric Clinical and Translational Research Center for their outstanding care of the participants and meticulous attention to the research, and to Resa Stauffer for all the laboratory analyses.

FUNDING This work was supported by NIH grants R01 HD-27503 (SA), K24 HD-01357 (SA), Richard L Day endowed Chair, and NIH/NCRR/CTSA UL1 RR024153

AUTHOR CONTRIBUTIONS SB analyzed these data and wrote the manuscript. SL, HT, FB, TM contributed participants and data and reviewed the manuscript. SL contributed technical analyses of body composition and abdominal adiposity. SA provided the study concept and design, acquired data, obtained funding, provided administrative, technical and material support, supervised the study and critically reviewed/edited the manuscript. 


\section{LIST OF ABBREVIATIONS}

$\begin{array}{ll}\text { BMI } & \text { body mass index } \\ \text { CV } & \text { coefficients of variation } \\ \text { DEXA } & \text { dual energy X-ray absorptiometry } \\ \text { HDL } & \text { high-density lipoprotein } \\ \text { HOMA-IR } & \text { Homeostasis Model Assessment- Insulin Resistance Index } \\ \text { ICAM-1 } & \text { intercellular adhesion molecule-1 } \\ \text { IFG } & \text { impaired fasting glucose } \\ \text { IGT } & \text { impaired glucose tolerance } \\ \text { LDL } & \text { low-density lipoprotein } \\ \text { NGT } & \text { normal glucose tolerance } \\ \text { NHANES } & \text { National Health and Nutrition Examination Survey } \\ \text { NMR } & \text { nuclear magnetic resonance } \\ \text { OGTT } & \text { oral glucose tolerance test } \\ \text { PD } & \text { pre-diabetes } \\ \text { TG } & \text { triglycerides } \\ \text { VAT } & \text { visceral adipose tissues } \\ \text { VCAM-1 } & \text { vascular adhesion molecule-1 } \\ \text { VLDL } & \text { very low-density lipoprotein }\end{array}$

\section{REFERENCES}

1. American Diabetes Association. Diagnosis and classification of diabetes mellitus. Diabetes Care. 2010; 33:S62-9. [PubMed: 20042775]

2. DeFronzo RA, Abdul-Ghani M. Assessment and treatment of cardiovascular risk in prediabetes: impaired glucose tolerance and impaired fasting glucose. Am J Cardiol. 2011; 108:3B-24B.

3. Li C, Ford ES, Zhao G, et al. Prevalence of pre-diabetes and its association with clustering of cardiometabolic risk factors and hyperinsulinemia among U.S. adolescents: National Health and Nutrition Examination Survey 2005-2006. Diabetes Care. 2009; 32:342-7. [PubMed: 18957533]

4. Sinha R, Fisch G, Teague B, et al. Prevalence of impaired glucose tolerance among children and adolescents with marked obesity. N Engl J Med. 2002; 346:802-10. [PubMed: 11893791]

5. Williams DE, Cadwell BL, Cheng YJ, et al. Prevalence of impaired fasting glucose and its relationship with cardiovascular disease risk factors in US adolescents, 1999-2000. Pediatrics. 2005; 116:1122-26. [PubMed: 16263998]

6. Packard CJ. LDL subfractions and atherogenicity: an hypothesis from the University of Glasgow. Curr Med Res Opin. 1996; 13:379-90. [PubMed: 8862937]

7. Blake GJ, Otvos JD, Rifai N, et al. Low-density lipoprotein particle concentration and size as determined by nuclear magnetic resonance spectroscopy as predictors of cardiovascular disease in women. Circulation. 2002; 106:1930-37. [PubMed: 12370215] 
8. Kuller L, Arnold A, Tracy R, et al. Nuclear magnetic resonance spectroscopy of lipoproteins and risk of coronary heart disease in the cardiovascular health study. Arterioscler Thromb Vasc Biol. 2002; 22:1175-80. [PubMed: 12117734]

9. Festa A, Williams K, Hanley AJ, et al. Nuclear magnetic resonance lipoprotein abnormalities in prediabetic subjects in the Insulin Resistance Atherosclerosis Study. Circulation. 2005; 111:346572. [PubMed: 15983261]

10. Wang J, Stančáková A, Soininen P, et al. Lipoprotein subclass profiles in individuals with varying degrees of glucose tolerance: a population-based study of 9399 Finnish men. J Intern Med. 2012; 272:562-72. [PubMed: 22650159]

11. Magge SN, Prasad D, Koren D, et al. Prediabetic obese adolescents have a more atherogenic lipoprotein profile compared with normoglycemic obese peers. J Pediatr. 2012; 161:881-6. [PubMed: 22622051]

12. Burns SF, Arslanian SA. Waist circumference, atherogenic lipoproteins, and vascular smooth muscle biomarkers in children. J Clin Endocrinol Metab. 2009; 94:4914-22. [PubMed: 19846736]

13. D'Adamo E, Northrup V, Weiss R, et al. Ethnic differences in lipoprotein subclasses in obese adolescents: importance of liver and intraabdominal fat accretion. Am J Clin Nutr. 2010; 92:5008. [PubMed: 20573788]

14. Ross R. Atherosclerosis--an inflammatory disease. N Engl J Med. 1999; 340:115-26. [PubMed: 9887164]

15. Arslanian SA, Saad R, Lewy V, et al. Hyperinsulinemia in african-american children: decreased insulin clearance and increased insulin secretion and its relationship to insulin sensitivity. Diabetes. 2002; 51:3014-19. [PubMed: 12351441]

16. Lee S, Gungor N, Bacha F, et al. Insulin resistance: link to the components of the metabolic syndrome and biomarkers of endothelial dysfunction in youth. Diabetes Care. 2007; 30:2091-97. [PubMed: 17475936]

17. Burns SF, Lee SJ, Arslanian SA. Surrogate lipid markers for small dense low-density lipoprotein particles in overweight youth. J Pediatr. 2012; 161:991-6. [PubMed: 22809659]

18. Burns SF, Lee S, Arslanian SA. In vivo insulin sensitivity and lipoprotein particle size and concentration in black and white children. Diabetes Care. 2009; 32:2087-93. [PubMed: 19675203]

19. Danadian K, Balasekaran G, Lewy V, et al. Insulin sensitivity in African-American children with and without family history of type 2 diabetes. Diabetes Care. 1999; 22:1325-9. [PubMed: 10480778]

20. Friedewald WT, Levy RI. Fredrickson DS Estimation of the concentration of low-density lipoprotein cholesterol in plasma, without use of the preparative ultracentrifuge. Clin Chem. 1972; 18:499-502. [PubMed: 4337382]

21. Otvos JD, Jeyarajah EJ, Bennett DW, et al. Development of a proton nuclear magnetic resonance spectroscopic method for determining plasma lipoprotein concentrations and subspecies distributions from a single, rapid measurement. Clin Chem. 1992; 38:1632-8. [PubMed: 1326420]

22. Jeyarajah EJ, Cromwell WC, Otvos JD. Lipoprotein particle analysis by nuclear magnetic resonance spectroscopy. Clin Lab Med. 2006; 26:847-70. [PubMed: 17110242]

23. Toledo-Corral CM, Vargas LG, Goran MI, et al. Hemoglobin A1c above threshold level is associated with decreased $\beta$-cell function in overweight Latino youth. J Pediatr. 2012; 160:751-6. [PubMed: 22137671]

24. The DECODE study group; European Diabetes Epidemiology Group. Diabetes Epidemiology: Collaborative analysis Of Diagnostic criteria in Europe. Glucose tolerance and mortality: comparison of WHO and American Diabetes Association diagnostic criteria. Lancet. 1999; 354:617-21. [PubMed: 10466661]

25. DECODE Study Group; European Diabetes Epidemiology Group. Glucose tolerance and cardiovascular mortality: comparison of fasting and 2-hour diagnostic criteria. Arch Intern Med. 2001; 161:397-405. [PubMed: 11176766]

26. Lehto S, Rönnemaa T, Haffner SM, et al. Dyslipidemia and hyperglycemia predict coronary heart disease events in middle-aged patients with NIDDM. Diabetes. 1997; 46:1354-9. [PubMed: 9231662] 
27. Coutinho M, Gerstein HC, Wang Y, et al. The relationship between glucose and incident cardiovascular events. A metaregression analysis of published data from 20 studies of 95,783 individuals followed for 12.4 years. Diabetes Care. 1999; 22:233-40. [PubMed: 10333939]

28. Ford ES, Zhao G, Li C. Pre-diabetes and the risk for cardiovascular disease: a systematic review of the evidence. J Am Coll Cardiol. 2010; 55:1310-7. [PubMed: 20338491]

29. Mora S, Szklo M, Otvos JD, et al. LDL particle subclasses, LDL particle size, and carotid atherosclerosis in the Multi-Ethnic Study of Atherosclerosis (MESA). Atherosclerosis. 2007; 192:211-7. [PubMed: 16765964]

30. Juonala M, Viikari JS, Kähönen M, et al. Life-time risk factors and progression of carotid atherosclerosis in young adults: the Cardiovascular Risk in Young Finns study. Eur Heart J. 2010; 31:1745-51. [PubMed: 20501481]

31. Li S, Chen W, Srinivasan SR, et al. Relation of childhood obesity/cardiometabolic phenotypes to adult cardiometabolic profile: the Bogalusa Heart Study. Am J Epidemiol. 2012; 176:S142-9. [PubMed: 23035138]

32. McGill HC Jr, McMahan CA, Zieske AW, et al. Associations of coronary heart disease risk factors with the intermediate lesion of atherosclerosis in youth. The Pathobiological Determinants of Atherosclerosis in Youth (PDAY) Research Group. Arterioscler Thromb Vasc Biol. 2000; 20:1998-2004. [PubMed: 10938023]

33. Davis PH, Dawson JD, Riley WA, et al. Carotid intimal-medial thickness is related to cardiovascular risk factors measured from childhood through middle age: The Muscatine Study. Circulation. 2001; 104:2815-9. [PubMed: 11733400]

34. Libman IM, Barinas-Mitchell E, Bartucci A, et al. Fasting and 2-hour plasma glucose and insulin: relationship with risk factors for cardiovascular disease in overweight nondiabetic children. Diabetes Care. 2010; 33:2674-6. [PubMed: 21115769]

35. Freedman DS, Bowman BA, Otvos JD, et al. Levels and correlates of LDL and VLDL particle sizes among children: the Bogalusa heart study. Atherosclerosis. 2000; 152:441-9. [PubMed: 10998473]

36. Pérez-Méndez O, Torres-Tamayo M, Posadas-Romero C, et al. Abnormal HDL subclasses distribution in overweight children with insulin resistance or type 2 diabetes mellitus. Clin Chim Acta. 2007; 376:17-22. [PubMed: 16934792]

37. TODAY Study Group. Lipid and inflammatory cardiovascular risk worsens over 3 years in youth with type 2 diabetes: the TODAY clinical trial. Diabetes Care. 2013; 36:1758-64. [PubMed: 23704675]

38. Strong JP, Malcom GT, McMahan CA, et al. Prevalence and extent of atherosclerosis in adolescents and young adults: implications for prevention from the Pathobiological Determinants of Atherosclerosis in Youth Study. JAMA. 1999; 281:727-35. [PubMed: 10052443]

39. Weiss TW, Arnesen H, Seljeflot I. Components of the interleukin-6 transsignalling system are associated with the metabolic syndrome, endothelial dysfunction and arterial stiffness. Metabolism. 2013; 62:1008-13. [PubMed: 23428306]

40. Yudkin JS, Montori VM. The epidemic of pre-diabetes: the medicine and the politics. BMJ. 2014; 15:g4485. 349. [PubMed: 25028385]

41. Abdul-Ghani MA, Jenkinson CP, Richardson DK, et al. Insulin secretion and action in subjects with impaired fasting glucose and impaired glucose tolerance: results from the Veterans Administration Genetic Epidemiology Study. Diabetes. 2006; 55:1430-35. [PubMed: 16644701]

42. Abdul-Ghani MA, Tripathy D, DeFronzo RA. Contributions of beta-cell dysfunction and insulin resistance to the pathogenesis of impaired glucose tolerance and impaired fasting glucose. Diabetes Care. 2006; 29:1130-39. [PubMed: 16644654]

43. Libman IM, Barinas-Mitchell E, Bartucci A, et al. Reproducibility of the oral glucose tolerance test in overweight children. J Clin Endocrinol Metab. 2008; 93:4231-7. [PubMed: 18713820]

44. Weiss R, Taksali SE, Tamborlane WV, et al. Predictors of changes in glucose tolerance status in obese youth. Diabetes Care. 2005; 28:902-9. [PubMed: 15793193]

45. Morris DH, Khunti K, Achana F, et al. Progression rates from HbA1c 6.0-6.4\% and other prediabetes definitions to type 2 diabetes: a meta-analysis. Diabetologia. 2013; 56:1489-93. [PubMed: 23584433] 
46. George L, Bacha F, Lee S, et al. Surrogate estimates of insulin sensitivity in obese youth along the spectrum of glucose tolerance from normal to prediabetes to diabetes. J Clin Endocrinol Metab. 2011; 96:2136-45. [PubMed: 21508130] 

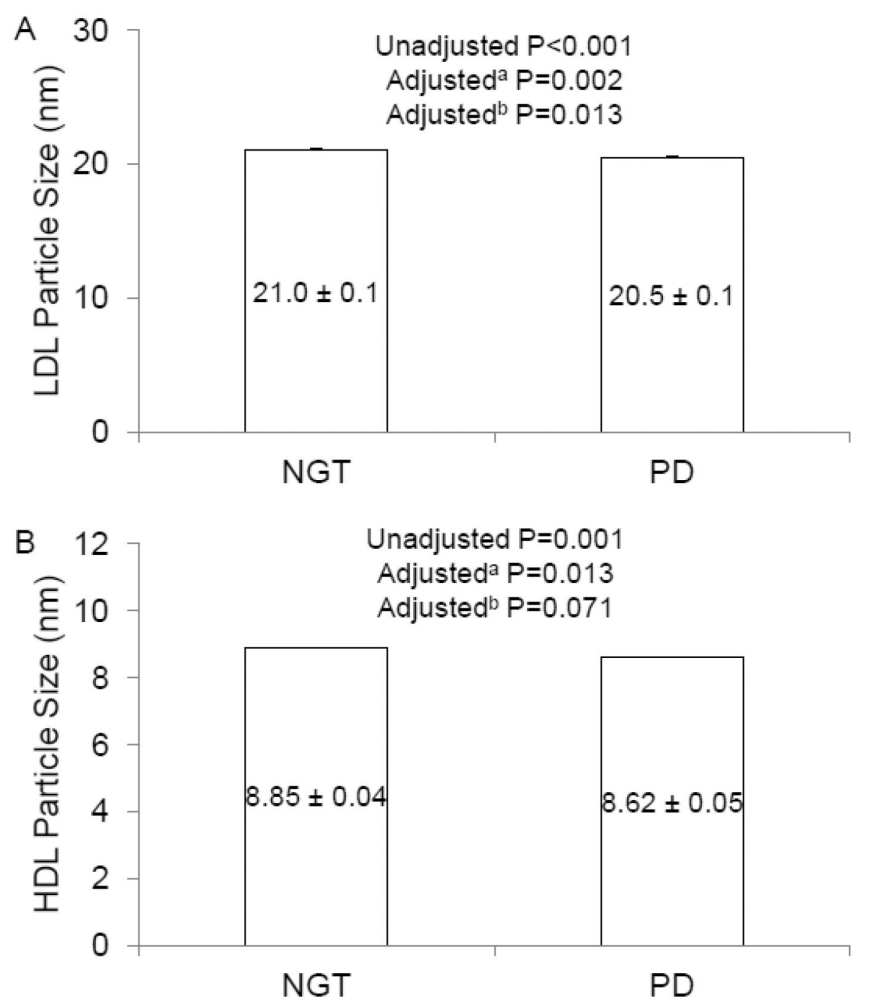

Figure 1.

LDL (panel A) and HDL (panel B) particle size in overweight/obese youth with normal glucose tolerance (NGT) and pre-diabetes (PD). Differences compared using an independent t-test or Mann Whitney test. Adjusted ${ }^{\mathrm{a}} \mathrm{P}$ is for the difference after adjusting for race and BMI using ANCOVA. Adjusted ${ }^{\mathrm{b}} \mathrm{P}$ is for the difference after adjusting for race, $\mathrm{BMI}$ and Log fasting insulin using ANCOVA. 

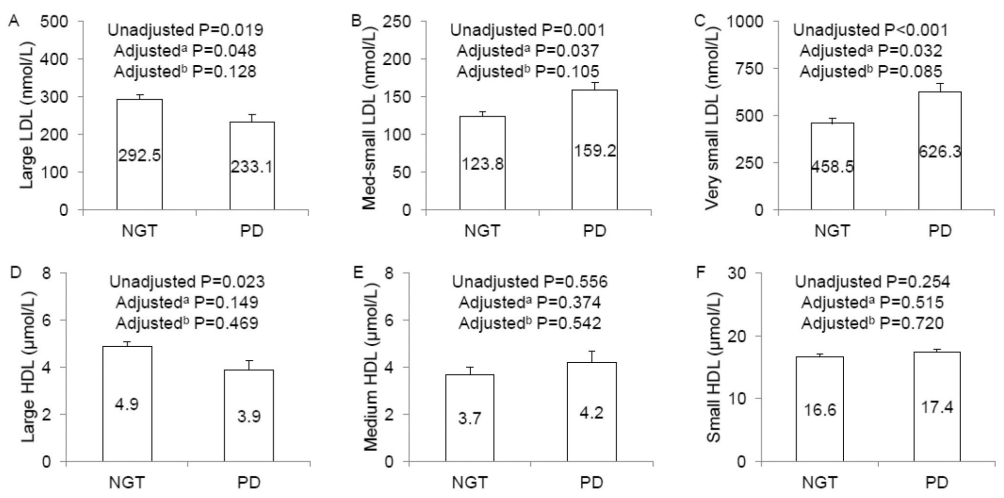

Figure 2.

Concentrations of large (panel A), medium-small (panel B) and very small (panel C) LDL particles, and large (panel D), medium (panel E) and small (panel F) HDL particles in youth with NGT and PD. Differences compared using an independent t-test or Mann Whitney test. Adjusted $^{\mathrm{a}} \mathrm{P}$ is for the difference after adjusting for race and BMI using ANCOVA. Adjusted $^{\mathrm{b}} \mathrm{P}$ is for the difference after adjusting for race, BMI and Log fasting insulin using ANCOVA. 


\section{Table 1}

Physical and metabolic characteristics, lipid profile and vascular smooth muscle markers of participants by category of glycemia (normal glucose tolerance, NGT and pre-diabetes, PD).

\begin{tabular}{|c|c|c|c|}
\hline & NGT (n=99) & PD $(n=45)$ & $\mathbf{P}$ \\
\hline \multicolumn{4}{|l|}{ Physical characteristics } \\
\hline Age (years) ${ }^{b}$ & $14.4 \pm 0.2$ & $14.7 \pm 0.3$ & 0.474 \\
\hline $\operatorname{Sex}(\mathrm{M} / \mathrm{F})^{a}$ & $30 / 69$ & $12 / 33$ & 0.656 \\
\hline $\operatorname{Race}(\mathrm{B} / \mathrm{W})^{a}$ & $50 / 49$ & $10 / 35$ & 0.001 \\
\hline Tanner stage $^{a}$ & & & 0.427 \\
\hline II-III & 21 & 7 & \\
\hline IV-V & 78 & 38 & \\
\hline $\operatorname{BMI}\left(\mathrm{kg} / \mathrm{m}^{2}\right)^{b}$ & $34.9 \pm 0.8$ & $36.1 \pm 1.0$ & 0.352 \\
\hline BMI percentile ${ }^{c}$ & $97.1 \pm 0.3$ & $98.3 \pm 0.2$ & 0.111 \\
\hline Body fat $(\%)^{c}$ & $42.7 \pm 0.8$ & $44.6 \pm 0.7$ & 0.471 \\
\hline Fat mass $(\mathrm{kg})^{b}$ & $39.1 \pm 1.4$ & $42.8 \pm 1.8$ & 0.132 \\
\hline $\operatorname{VAT}\left(\mathrm{cm}^{2}\right)^{c}$ & $63.7 \pm 4.4$ & $81.1 \pm 5.7$ & 0.001 \\
\hline \multicolumn{4}{|l|}{ Metabolic characteristics } \\
\hline Fasting glucose $(\mathrm{mmol} / \mathrm{L})^{b}$ & $4.87 \pm 0.04$ & $5.25 \pm 0.07$ & $<0.001$ \\
\hline 2-h glucose (OGTT) $(\mathrm{mmol} / \mathrm{L})^{c}$ & $6.41 \pm 0.08$ & $8.51 \pm 0.19$ & $<0.001$ \\
\hline $\mathrm{HbA}_{1 \mathrm{c}}(\%)$ & $5.3 \pm 0.0$ & $5.3 \pm 0.1$ & 0.430 \\
\hline$(\mathrm{mmol} / \mathrm{mol})^{c}$ & $(34.0 \pm 1.4)$ & $(34.0 \pm 2.0)$ & \\
\hline Fasting insulin $(\mathrm{pmol} / \mathrm{L})^{c}$ & $196.8 \pm 16.8$ & $234.0 \pm 23.4$ & 0.027 \\
\hline \multicolumn{4}{|l|}{ Fasting lipid profile } \\
\hline Total cholesterol $(\mathrm{mmol} / \mathrm{L})^{b}$ & $4.16 \pm 0.10$ & $4.24 \pm 0.12$ & 0.640 \\
\hline HDL-cholesterol $(\mathrm{mmol} / \mathrm{L})^{c}$ & $1.38 \pm 0.07$ & $1.04 \pm 0.05$ & 0.001 \\
\hline LDL-cholesterol $(\mathrm{mmol} / \mathrm{L})^{b}$ & $2.24 \pm 0.10$ & $2.48 \pm 0.12$ & 0.138 \\
\hline $\mathrm{TG}(\mathrm{mmol} / \mathrm{L})^{c}$ & $1.22 \pm 0.06$ & $1.55 \pm 0.12$ & 0.013 \\
\hline $\operatorname{VLDL}-\mathrm{TG}(\mathrm{mmol} / \mathrm{L})^{c}$ & $0.23 \pm 0.01$ & $0.31 \pm 0.02$ & 0.004 \\
\hline \multicolumn{4}{|l|}{ Vascular smooth muscle markers } \\
\hline ICAM-1 (ng/mL) ${ }^{c}$ & $222.5 \pm 9.9$ & $231.9 \pm 17.0$ & 0.852 \\
\hline $\mathrm{VCAM}-1(\mathrm{ng} / \mathrm{mL})^{c}$ & $683.6 \pm 26.1$ & $722.8 \pm 43.9$ & 0.498 \\
\hline E-selectin $(\mathrm{ng} / \mathrm{mL})^{c}$ & $53.6 \pm 3.6$ & $48.7 \pm 4.0$ & 0.595 \\
\hline
\end{tabular}

Metabolism. Author manuscript; available in PMC 2015 December 01. 
${ }^{b}$ Compared using independent t-test

${ }^{c}$ Compared using Mann Whitney test 


\section{Table 2}

Stepwise multiple linear regression to quantify the independent contribution of category of glycemia [(normal glucose tolerance (NGT) and pre-diabetes (PD)], race, sex, age, Tanner stage, BMI and fasting insulin to LDL particle size and concentration.

\begin{tabular}{|c|c|c|c|c|}
\hline Dependent variable & Independent variables ${ }^{a}$ & Partial $\mathbf{r}$ & $\mathbf{P}$ & $\mathbf{R}^{2}$ \\
\hline \multirow[t]{7}{*}{ LDL Size } & Glucose category & -0.218 & 0.010 & \\
\hline & Race & -0.173 & 0.042 & \\
\hline & Sex & 0.205 & 0.016 & \\
\hline & Age & 0.054 & 0.529 & \\
\hline & Tanner stage & 0.104 & 0.223 & \\
\hline & BMI & -0.031 & 0.715 & \\
\hline & Log fasting insulin & -0.344 & $<0.001$ & 0.243 \\
\hline \multirow[t]{7}{*}{ Medium small LDL concentration } & Glucose category & 0.170 & 0.045 & \\
\hline & Race & 0.082 & 0.335 & \\
\hline & Sex & -0.184 & 0.029 & \\
\hline & Age & -0.023 & 0.787 & \\
\hline & Tanner stage & -0.075 & 0.383 & \\
\hline & BMI & 0.033 & 0.700 & \\
\hline & Log fasting insulin & 0.356 & $<0.001$ & 0.184 \\
\hline \multirow[t]{7}{*}{ Very small LDL concentration } & Glucose category & 0.182 & 0.031 & \\
\hline & Race & 0.101 & 0.235 & \\
\hline & Sex & -0.171 & 0.043 & \\
\hline & Age & -0.056 & 0.512 & \\
\hline & Tanner stage & -0.068 & 0.425 & \\
\hline & BMI & 0.058 & 0.500 & \\
\hline & Log fasting insulin & 0.352 & $<0.001$ & 0.183 \\
\hline
\end{tabular}

${ }^{a}$ Final model 